\title{
Connectivity-Based Parcellation of Functional SubROIs in Putamen using A Sparse Spatially Regularized Regression Model
}

\author{
Yiming Zhang ${ }^{\mathrm{a}}$, Aiping $\mathrm{Liu}^{\mathrm{c}, *}$, Sun Nee Tan ${ }^{\mathrm{b}}$, Martin J. McKeown ${ }^{\mathrm{b}}$, Z. Jane \\ Wanga \\ ${ }^{a}$ Department of Electrical and Computer Engineering, \\ University of British Columbia, V6T 1Z4, Vancouver, Canada \\ ${ }^{b}$ Department of Neurology and Pacific Parkinson's Research Centre, \\ University of British Columbia, V6T 1Z4, Vancouver, Canada \\ ${ }^{c}$ Department of Biomedical Engineering, \\ Hefei University of Technology, 193 Tunxi Road, 230009 Hefei,China
}

\begin{abstract}
In this paper, we present a novel framework for parcellation of a brain region into functional subROIs (Sub-Region-of-Interest) based on their connectivity patterns with other brain regions. By utilising previously established neuroanatomy information, the proposed method aims at finding spatially-continuous, functionally consistent subROIs in a given brain region. The proposed framework relies on 1) a sparse spatially-regularized fused lasso regression model for encouraging spatially and functionally adjacent voxels to share similar regression coefficients; 2) an iterative merging and adaptive parameter tuning process; 3) a Graph-Cut optimization algorithm for assigning overlapped voxels into separate subROIs. Our simulation results demonstrate that the proposed method could reliably yield spatially continuous and functionally consistent subROIs. We applied the method to resting-state fMRI data obtained from normal subjects and explored connectivity to the putamen. Two distinct functional subROIs could be parcellated out in the putamen region in all subjects. This approach provides a way to extract functional subROIs that can then be investigated for alterations in connectivity in diseases of the basal ganglia, for example in
\end{abstract}

\footnotetext{
* Corresponding author

Email address: aipingl@ece.ubc.ca (Aiping Liu )
}

Preprint submitted to Biomedical Signal Processing and Control

October 18, 2016 
Parkinson's disease.

Keywords: fMRI, brain connectivity, brain parcellation, spatial regularization

\section{Introduction}

Functional magnetic resonance imaging (fMRI) is a functional neuroimaging technique that indirectly measures brain activity by detecting associated alterations in blood oxygenation (BOLD signal). In the past, most fMRI studies focused on detection of localized neural activities by modeling the relationship between fMRI signals and experiment stimulus, i.e., activity studies 1, 2, 3. However, the human brain relies on efficient networks of interacting brain regions [4. Hence, interests in studying the associations between brain regions have grown, i.e., connectivity studies [5, 6, 7]. Connectivity studies can be explored using task-related as well as resting state fMRI data, with the latter looking at spontaneous interactions between different brain regions without requiring active engagement from the subject. Resting state fMRI may therefore be more suitable for studies involving aging and diseased populations [8] who oftentimes suffer from sensory, motor and/or cognitive impairments rendering them incapable of performing challenging tasks.

Connectivity studies can be conducted at either the voxel or ROI (regionsof-interest) level. Voxel-based approaches usually involve a large number of variables, are computationally-inefficient, and must account for the massive amount of multiple comparisons. Such voxel-based approaches are typically done by spatially transforming all brain volumes to the same anatomical template. However, subtle misregistration can make the assumption that, after registration, a given voxel will represent the same functional region across all subjects tenuous. ROI-based connectivity analysis may reduce the number of multiple comparisons, and does not necessarily require spatial transformation, but requires careful consideration as to the definition of an ROI. Anatomical ROIs may be used to infer functional ROIs [9, but a single anatomical ROI, such as the putamen or amygdala, may in fact encompass distinct functional subROIs 


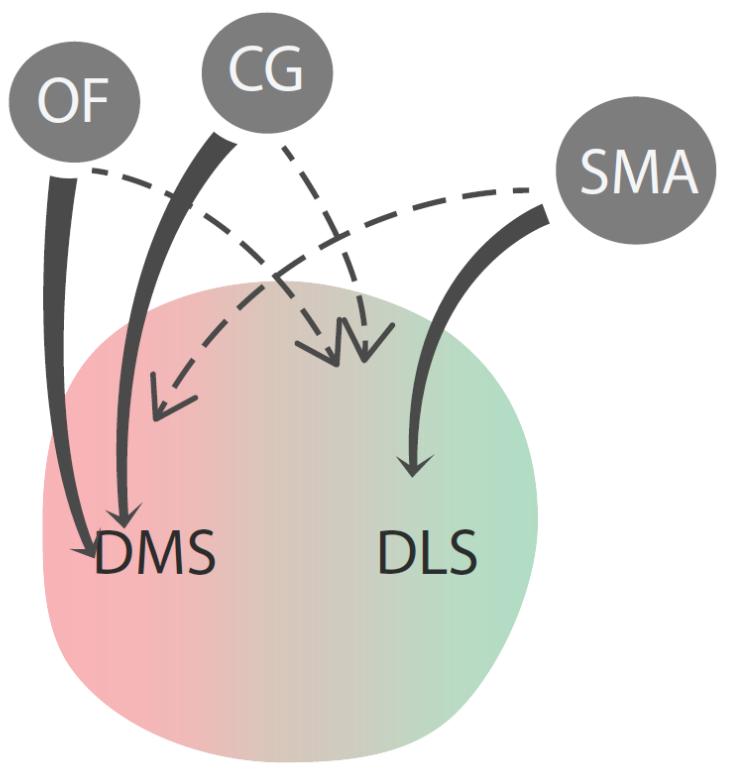

Figure 1: Illustration of functional subROIs in the putamen brain region. The figure is made according to the graph shown in 18 .

[10. A number of attempts have been made to utilize data-driven approaches to subdivide a given ROIs into subROIs based on functional connectivity. One broad approach is based on cluster analysis [1] which first retrieves connectivity features of each voxel within an ROI using general linear regression models or Pearson's pairwise correlation coefficients, and then applies clustering according to the functional distances defined by the extracted features. Various clustering methods have been adopted in previous studies, such as the fuzzy clustering method [11, k-means clustering method [12, a self-organized mapping method [10] and maximum margin clustering method [13]. However, in order to acquire spatially continuous results, most clustering methods require meticulous denoising preprocessing methods as they are very sensitive to outliers in the data. Another popular category of data-driven approaches is based on graph theory, where each voxel represents one node in the graph, and methods such as the normalized cut approach [14] and modularity detection method [15] are used to separate the graph (and hence ROI) into distinct subROIs. Similar to clustering 
methods, graph theory methods do not take into account spatial information, and thus it is often difficult to obtain spatially continuous subROIs using graph theory methods. Furthermore, most graph theory methods are only concerned with the connectivity map between voxels within an ROI without incorporating connectivity from other ROIs, which might limit their usefulness.

In this paper, we propose a novel framework which defines subROIs in one ROI based on their functional connectivity to other ROIs. The proposed method employs a fused lasso regression model [16] with a spatial regularization penalty incorporated. The fused lasso approach encourages sparsity of the regression coefficients as well as sparsity of their successive differences between coefficients. We further introduce the normal lasso penalty for all voxels and the fused lasso penalty on spatially adjacent pairs of voxels.

Our framework differentiates from other approaches in the literature [1] [12] [13 14 15] in two main aspects. Firstly, in our proposed framework, we utilize the functional connectivity between voxels in the task ROI and the average time series of other related ROIs (where the task ROI and the related ROIs belong to one neural control loop). In the literature [11 14] 15, people only consider the functional connectivity between voxels within the task ROI. Secondly, we incorporate spatial information that is often ignored in the literature [11] 14] 15] into our problem formulation. In addition, in order to limit the amount of bias that spatial constraint could introduce, we proposed a novel algorithm for adaptive, data-dependent parameter selection which allows us to only add spatial constraint when it is 'necessary'.

Functionally, in the basal ganglia-cortical loops in animals, the putamen is connected to several cortices with a clear topography [18. We have chosen three reference brain regions namely the orbitofrontal (OF) cortex, cingulate gyrus (CG) and sensorimotor cortex(SMA) to assess connectivity to the ROI of interest, the putamen. Specifically, the DLS is more strongly connected to the SMA while the DMS has more reciprocal connections with the OF and CG [19] [18. However, the spatial boundary between the DLS and the DMS is blurry and their exact locations are unknown due to overlapped connections; Some voxels 
of the DMS also have weak connections with the SMA while the DLS, too, may receive weak connections from the $\mathrm{CG}$ and $\mathrm{OF}$. This scenario is illustrated in Figure 1. In addition, as we observed in real fMRI data, due to the spatial signal noise, head movement and other possible artifacts, the data could be spatially corrupted with some outlier voxels. As a result, many current parcellation methods are not able to deal with such corrupted voxels to obtain a spatially continuous parcellation. Therefore, we plan to design an algorithm to parcellate the putamen region not only according to functional connectivity features from prior knowledge but also through integration of spatial information.

This pilot study aimed to investigate a novel technique to parcellate the putamen into two subregions with distinct functional and structural connections to the cortices of the brain. The putamen and caudate are two structurally distinct brain regions in the brainstem with the former lying more laterally and inferior to the latter. Due to the proximity of these two brain regions to each other and their shared neuronal connections, together, they are known as the striatum. Within the striatum (caudate and putamen), it has spatially segregated functional topography. The dorsolateral striatum (DLS) consists of the dorsal and lateral aspects of the caudate and putamen, and is associated with control of habitual, automatic movements. On the other hand, more medial areas within the caudate and putamen are known as the dorsomedial striatum (DMS) and this region is functionally related to learning and execution of goaloriented movements. As an exploratory first step, in this paper, we chose to parcellate the putamen into the DLS and DMS subregions.

In the remainder of the paper, we will present the proposed method in Section 2. In Section 3.1. we investigated on a synthetic dataset to compare the results of the proposed method with those of clustering and graph theory methods. We tested the proposed method on real resting state fMRI data set in Section 3.2. In Section 4 , we presented a summary of our results with a conclusion. 


\section{Method}

In this section, we will describe the proposed framework to separate a given ROI into functionally consistent and spatially continuous subROIs. We define the ROI to be separated as the task ROI and other ROIs used to estimate the connections with the task ROI as reference ROIs. We intended to find subsets of adjacent voxels (i.e., subROIs) in the task ROI that share similar connectivity patterns to other reference ROIs and iteratively merge them into groups. In the proposed algorithm, according to prior neuroanatomical knowledge, there are several reference ROIs that share similar connectivity patterns with one functional subROI in the task ROI (putamen in this paper) so that we can obtain the functional boundary between these subROIs.

The proposed framework can be summarized in Table 1. We will now elaborate on the individual components of the proposed framework in the following subsections. First, we start by describing the spatially regularized fused lasso model.

\subsection{Spatially Regularized Fused Lasso Method}

Let $X=\left[x_{1}, x_{2}, \cdots, x_{n}\right]$ be a $(T \times n)$-dimensional data matrix with $n$ denoting the number of voxels and $T$ denoting the length of time points. $X$ represents the fMRI signals in the task ROI and $x_{i}, i=1,2, \cdots, n$ represents fMRI time course of voxel $i$. $Y$ is a $(T \times 1)$ vector representing the signals of one reference ROI which is acquired by averaging time courses across all voxels contained in that reference ROI. Let $\beta$ be a $(n \times 1)$ vector where each element in $\beta$ represents the connectivity coefficient between one voxel and the reference ROI. A standard linear model with one reference ROI $Y$ is:

$$
Y=X \beta+\epsilon
$$

with error $\epsilon$ assumed to be zero mean and constant variance.

The linear regression model tries to obtain the weight for each voxel in the task ROI in order to get the best linear fit for the reference signal, i.e., smallest 
Table 1: Connectivity-based Brain Parcellation Framework.

Input : $\quad X: T \times n$ matrix represents task ROI signal.

$Y_{1}, Y_{2}, \ldots, Y_{m}$ : Each $Y_{i}$ is a $T \times 1$ vector representing time course for reference ROI $i$

Step 1 : According to each reference ROI $Y_{i}$, iterate until final separation being obtained.

1) Perform spatially regularized fused lasso algorithm between voxels in the task ROI and one reference ROI.

2) Merge adjacent voxels with similar connectivity weight acquired from 1) into one group.

3) Treat groups from 2) as new voxels, go back to 1)

Step 2 : With more than one reference ROI, combine all parcellation results from all reference ROIs by performing Graph-Cut algorithm.

Output : Two spatially continuous functional subROIs from $X$

mean square error. So the problem could be formed as,

$$
\hat{\beta}=\arg \min _{\beta}\|X \beta-Y\|_{2}
$$

For fMRI data, it is common that the number of voxels $n$ in task ROI is larger than the length of time points $T$. In this case, the problem of multicollinearity will arise, and an optimal solution to above problem will not be unique. In order to eliminate the effect of multicollinearity and make the solution unique, we introduce the normal Lasso (Least Absolute Shrinkage and Selection Operator) penalty $\sum_{j=1}^{n}\left|\beta_{j}\right| \leq s_{1}$ where $s_{1}$ is a tuning parameter [20]. This penalty will encourage sparsity in the coefficient vector $\beta$, forcing small elements to be exactly zero. In addition, it is well known in fMRI analysis that similar connectivity patterns tend to exist in clusters of spatially adjacent voxels rather than small isolated groups of voxels [21]. However, with only the Lasso penalty, we cannot incorporate spatial information. Therefore, we further introduce a 
spatially regularized fused Lasso penalty $\sum_{j=1}^{n} \sum_{j<i} C_{j i}\left|\beta_{j}-\beta_{i}\right|<s_{2}$, where $s_{2}$ is a tuning parameter and

$$
C_{j i}= \begin{cases}1, & \text { if voxel } j \text { and } i \text { are adjacent } \\ 0, & \text { otherwise }\end{cases}
$$

This is a spatially regularized fused lasso penalty that comes from the normal fused lasso penalty [16], and it is also a special case of a graph-guided fused lasso model [22]. The spatially regularized fused lasso penalty will encourage sparsity in the difference between spatially adjacent voxels, forcing adjacent voxels to belong to the same cluster.

With the Lagrange multipliers' method, we could form the optimization problem of the spatially regularized fused lasso model as,

$$
\arg \min _{\beta}\left\{\|X \beta-Y\|_{2}+\lambda \sum_{j=1}^{n}\left|\beta_{j}\right|+\gamma \sum_{j=1}^{n} \sum_{j<i} C_{j i}\left|\beta_{j}-\beta_{i}\right|\right\}
$$

where the second term encourages sparsity among elements in $\beta$, and the third term encourages sparsity in differences between spatially successive elements.

In fMRI, usually $n>T$, i.e., the number of voxels within a ROI, $n$, is larger than the number of fMRI time points $T$. However, regarding to different sizes of ROIs and lengths of fMRI experiments, it is possible that in some ROIs we can have $n<T$. For instance, in the fMRI data we have been working on, the length of fMRI time course $\mathrm{T}(T=240)$ is larger than the number of voxels $n$ ( $n$ is around 200) in ROI Caudate. It is commonly recognized that if $n<T$ and if time courses of different voxels are independent to each other, the introduction of Lasso penalty to the regression model is redundant. However, according to realistic assumptions of fMRI data, the fMRI time series of different voxels within one ROI are not independent and indeed neighbour voxels are generally expected to have high correlations. Thus, many predictors suffer from collinearity which would allow the coefficients of predictors to be sparse and coefficient differences between adjacent voxels to be sparse. Therefore, the introduction of Lasso penalty and fused-lasso penalty is not unnecessary and the proposed algorithm is still feasible when $n<T$. 
Equation (4) leads to a quadratic programming problem with non-smooth constraints. In order to solve this problem efficiently, we implemented a Smoothing Proximal Gradient (SPG) method recently proposed [23].

\subsection{Merge Voxels into Groups}

With the lasso penalty and spatially regularized fused lasso penalty, the inferred connectivity weights $\beta$ and their differences should be sparse. In the second step, we merged adjacent voxels that share similar connectivity weights into groups. To better illustrate our method, we utilize some definitions from graph theory in our description. We mapped the task ROI as a connected graph, where each voxel is taken as one node in the graph, and the edge between two nodes exists only when these two nodes are adjacent. In this network, adjacency is defined by any pairs of nodes that are horizontally, vertically, or diagonally adjacent in a 3-dimensional space, i.e., the Euclidean distance between the two nodes is not greater than $\sqrt{3}$. We then merged those adjacent nodes corresponding to the same connectivity weights into the same group.

After acquiring new groups, we averaged the signals contained in each group and treated the averaged signals as new predictor variables. Then, we recalculated the connectivity weights of the updated predictor variables using the spatially regularized fused lasso model. After merging of the voxels (groups), edges in the updated graph exist only when the minimal distance between vox-

els in the two groups is not greater than $\sqrt{3}$. This process is repeated until the number of groups reaches the expected number of subROIs, which is 2 in our case. However, with real fMRI data, noise cannot be ignored and tuning parameters would also affect the results such that the algorithm may not converge. Therefore, if the number of groups remains unchanged after one iteration, we will increase the penalty parameters to enforce the regularization and relax the merging precision. Merging precision is controlled by a rounding parameter $\delta$ which is defined as the precision of judging the equality of two connectivity weights (For instance, merging precision $\delta=10^{4}$ means that the connectivity weights will be compared by 4 digits after the decimal point). 
The detailed algorithm of parcellation with one reference ROI is described in Algorithm 1

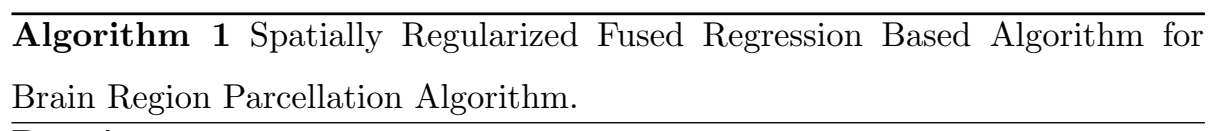

\section{Require:}

task ROI voxel level signal $X$, the mean time course $Y$ of the reference ROI, the lasso penalty parameter $\lambda$, the fused lasso penalty parameter $\gamma$ and the merging precision parameter $\delta$.

\section{Initialization:}

(1)Run spatially regularized fused regression model on all voxels in task ROI, get connectivity weights $\beta$ for all voxels.

(2)Round $\beta$ by $\delta$, merge adjacent voxels with same connectivity weights into one group, get $m$ groups. Before iteration, save original merging precision parameter, $\delta_{\text {initial }} \leftarrow \delta$.

\section{Ensure:}

1: while $m \neq 2$ do

2: Take groups as nodes, use mean value to represent groups signal. Run spatial regularized fused lasso regression on all nodes;

3: $\quad$ Round $\beta$ by $\delta$, merge adjacent nodes who have same connectivity weights into one group, get $m$ groups.

4: $\quad$ if $m=1$ then

5: $\quad \gamma \leftarrow \gamma / 2, \lambda \leftarrow \lambda / 2, \delta \leftarrow \delta \times 10$, redo 2 and 3 ;

6: $\quad$ else

7: $\quad$ if $m$ remain unchanged then

8: $\quad \gamma \leftarrow \gamma+1, \lambda \leftarrow \lambda+1, \delta \leftarrow \delta / 10$, redo 2 and 3 ;

9: $\quad$ end if

10: else

11: $\quad \delta \leftarrow \delta_{\text {initial }}$, continue

12: $\quad$ end if

13: end while 


\subsection{Assign Voxels Using the Graph-Cut Optimization}

In our study, three reference ROIs were used for parcellation of the task ROI, i.e., the putamen. After obtaining parcellation based on each reference ROI respectively, there were some overlapped voxels with different group assignments around the boundaries of all parcellation results. In order to further define the boundary between the DLS and DMS, we combined all information from the reference ROIs, and applied the Graph-Cut algorithm to assign those voxels into two groups.

The Graph-Cut algorithm is mostly applied in the field of computer vision, where it could be used for image smoothing, segmentation, and many other computer vision problems which could be categorised under energy minimization problems. It is basically a combinatorial optimization technique based on network flow theory (i.e., max-flow min-cut theory [24]). Once an energy minimization problem is formulated in terms of a network flow problem, many energy functions can be solved through the Graph-Cut algorithm.

In our framework, there were two determined subROIs and several voxels around the boundary to be assigned, so our goal is to assign those voxels into two groups. Firstly, we formalized the voxel assignment problem as the minimization of the following energy function,

$$
\min _{f} E(f)=\sum_{v} D_{v}\left(f_{v}\right)+\sum_{\{v, q\} \in N} V\left(f_{v}, f_{q}\right)
$$

where $f$ represents labels for all voxels, $f_{v}$ is the label of voxel $v, \sum_{v} D_{v}\left(f_{v}\right)$ is data energy which measures the cost of labeling voxel $v$ as $f_{v}, \sum_{\{v, q\} \in N} V\left(f_{v}, f_{q}\right)$ is the smooth energy that measures the extent to which the labeling $f$ is not piecewise smooth and $N$ is the set of all pairs of adjacent voxels. In summary, the first term in equation (5) tries to ensure that each voxel has been labeled correctly while the second term maintains the continuity of parcellation. Secondly, in order to solve the above energy minimization problem, we have to formulate the problem as a network flow problem and define $D_{v}\left(f_{v}\right), V\left(f_{v}, f_{q}\right)$. Here we construct the voxel assignment problem as network flow problem based on 25]. 


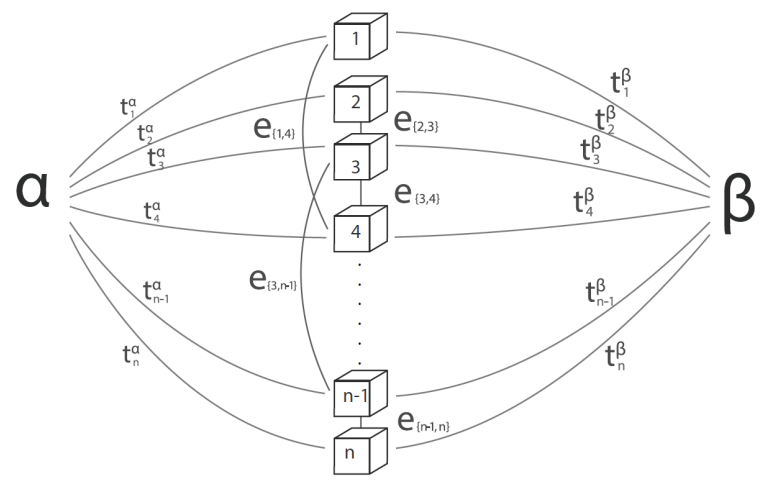

Figure 2: Illustration of graph example in the voxel assignment problem, where each small cube represents one voxel in an overlapped part of the task ROI. $\alpha$ and $\beta$ are labels of two determined subROIs in the task ROI. Link $e_{i, j}$ between voxels exist if voxel $i$ and voxel $j$ are neighbours. $t_{i}^{\alpha}$ and $t_{i}^{\beta}$ represent links between voxel $i$ and determined subROI $\alpha$ and $\beta$ respectively.

and solve it using the $\alpha \beta$-swap algorithm described in [26]. In our application of Graph-Cut, we define,

$$
\begin{gathered}
D_{v}\left(f_{v}\right)=1-\rho\left(v, f_{v}\right) \\
V(\alpha, \beta)=T(\alpha \neq \beta)
\end{gathered}
$$

where $\rho\left(v, f_{v}\right)$ is the Pearson's correlation between voxel $v$ and region $f_{v}$, region signal is calculated as mean time course of the obtained subROI $f_{v}$, and $T($.$) is$ Potts model.

In the $\alpha \beta$-swap algorithm, initial label $f$ must be given. Here we initialize the label $f_{v}$ for voxel $v$ as $\alpha$ if the number of voxel $v$ labeled as $\alpha\left(n_{\alpha}\right)$ is larger than the number of that labeled as $\beta\left(n_{\beta}\right)$, vice versa. If $n_{\alpha}=n_{\beta}$, we set the initial label randomly. Then we try to find a labeling $\hat{f}$ that minimize equation (5) over all labeling by swapping the labels for all voxels one by one. As described in Figure 2, let $P_{\alpha \beta}$ be the set of voxels to be assigned. There are two links connecting each voxel with terminal $\alpha$ and $\beta$, noted as $t_{p}^{\alpha}$ and $t_{p}^{\beta}$ where $p$ indicates the voxel $p$. There is another possible link connecting adjacent 
voxels $p$ and $q$ together, noted as $e_{\{p, q\}}$. According to [25], the weights assigned to the links are,

$$
\begin{aligned}
& t_{p}^{\alpha}=D_{p}(\alpha)+\sum_{q \in N_{v}, q \notin P_{\alpha \beta}} V\left(\alpha, f_{q}\right) \quad \text { for } v \in P_{\alpha \beta} \\
& t_{p}^{\beta}=D_{p}(\beta)+\sum_{q \in N_{v}, q \notin P_{\alpha \beta}} V\left(\beta, f_{q}\right) \quad \text { for } v \in P_{\alpha \beta} \\
& e_{\{p, q\}}=V(\alpha, \beta) \quad \text { for }\{v, q\} \in N \text { and } v, q \in P_{\alpha \beta}
\end{aligned}
$$

where $N_{v}$ is the set of adjacent voxels of $v, N$ is the set of all pairs of adjacent voxel. Therefore, the energy function (5) can be solved through maximum-flow algorithm on this graph [27.

\subsection{Extension to Regions with Three or More SubROIs}

Although the aforementioned algorithm was applied to our fMRI dataset where the task ROI is assumed to have two subROIs, the method can be easily extended to other brain regions that may contain three or more subROIs, with some small modifications: First, for the parcellation of the task ROI according to one reference ROI, we only need to modify the stop criteria. The algorithm will stop merging groups when the number of groups reaches the expected number of subROIs. Second, the Graph-Cut approach is still applicable in this case. In the case with two subROIs, the $\alpha \beta$ swap algorithm only applies on one pair of labels, while in any case with three or more subROIs, the $\alpha \beta$ swap algorithm needs to run on all pairs of labels. For each pair, the process is exactly the same as described earlier.

\section{Results}

\subsection{Synthetic Data Set}

In this section, we first tested the proposed method on synthetic datasets by comparing its performance with those of k-means clustering as well as the modularity detection approach. 


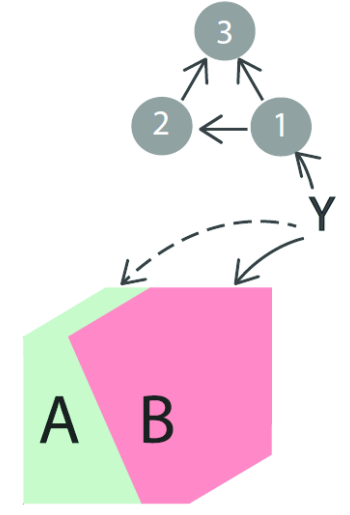

$$
\begin{gathered}
Y_{1}=\theta_{1} Y+\epsilon_{1} \\
Y_{2}=\theta_{2} Y_{1}+\epsilon_{2} \\
Y_{3}=\theta_{3}\left(Y_{1}+Y_{2}\right)+\epsilon_{3} \\
X_{A}=\alpha Y+(1-\alpha) Z+\epsilon_{A} \\
X_{B}=\beta Y+\epsilon_{B} \\
\epsilon_{1}, \epsilon_{2}, \epsilon_{3}, \epsilon_{A}, \epsilon_{B} \sim N\left(0, \sigma^{2}\right) \\
\theta_{1}, \theta_{2}, \theta_{3} \sim U[0.6,1]
\end{gathered}
$$

Figure 3: Illustration of the synthetic data generation. 1,2,3 indicate three connected reference ROIs. $Y_{1}, Y_{2}, Y_{3}$ represent signals in reference ROIs $1,2,3$ respectively. $X_{A}$ represents signal in region A. $X_{B}$ represents signal in region B. $Y$ and $Z$ are source signals generated from normal distribution with zero mean and unit variance. In order to comply with real fMRI signal, we further perform temporal Gaussian smoother on the source signal. Here we set $\beta=1$ and $\alpha=0.2$.

In order to ensure our simulations are compliant with real fMRI conditions, we generated three reference ROIs which are connected to each other and one cubical task ROI in which one spatially continuous subROI had strong connectivity to all three reference ROIs and the other part had weak connections with all reference ROIs. The boundary was set to separate these two subROIs. The process of generating the synthetic dataset was shown in Figure 3 , The $10 \times 10 \times 10$ cubic task ROI contains 1000 voxels, and the sample length of each voxel was 300. Similarly, each reference ROI contained 300 voxels with data length of 300 .

In the simulation, two different scenarios were considered. In the first case, the dataset was generated with the same signal to noise ratio (SNR) for all voxels (syn-data 1 ). Here $\mathrm{SNR}=\sigma_{\text {signal }}^{2} / \sigma_{\text {noise }}^{2}$ where $\sigma^{2}$ was the variance of the data. In the second simulation, we randomly chose 75 voxels in each subROI of the task ROI in synthetic data set 1 . Then, we assigned those voxels with smaller SNR, i.e., partially corrupted by outliers (syn-data 2 ). In order to ensure 
the boundary is clear in synthetic dataset 2 , we chose the outlier voxels that are away from the boundary. Results from the two datasets were compared with those of the k-means clustering method and the modularity detection method. Each procedure was repeated fifty times and the averaged performance of each algorithm was evaluated by looking at its error percentage, which is defined as,

$$
\text { Error Percentage }=\frac{\# \text { of all negative assigned voxels }}{\# \text { of all voxels in task ROI }}
$$

To implement the k-means algorithm according to [12, we first computed the Pearson's correlation coefficients between the task ROI and each reference ROI at the voxel level. Then, we transformed the Pearson's correlation $\rho$ into Fisher's $z$ statistics,

$$
z=0.5 \times \log \frac{1+\rho}{1-\rho}
$$

Then, each voxel of the task ROI will have a corresponding feature vector where each element represents the $z$ score between current voxel and one voxel in a reference ROI. $k$ was set to 2 and Euclidean distance measure was chosen in the simulation. For modularity detection method in [15, we constructed the undirected weighted graph by considering all voxels as nodes and their pairwise Pearson's correlation coefficients as edge weights. Since all algorithms perform well on syn-data 1 , in this paper, we only demonstrated one representative result from syn-data 2 in Figure 4

In addition, we performed k-means clustering with spatial regularization to further compare with our spatially regularized algorithm. In this paper, according to [28, we perform the spatially regularized k-means clustering method by extending the feature vector of each voxel in task ROI with three spatial coordinates, multiplied with a parameter $p$ indicating the weights given to the spatial information. Figure 5 shows the performance of algorithm on syn-data2 with different values of parameter $p$, form which we could infer that with spatial information incorporated result could be improved. However, the choice of parameter $p$ is crucial and a larger value would cause loss of robustness.

From the results, we noted that, without the incorporation of spatial infor- 
Table 2: Synthetic data sets

\begin{tabular}{lll}
\hline & SNR & Configuration \\
\hline syn-data1 & 0.5 & normal spatial noise \\
syn-data2 & $0.5($ full $)+0.1($ part $)$ & data with few outliers \\
\hline
\end{tabular}

Table 3: Best error percentage from synthetics data sets

\begin{tabular}{lll}
\hline & syn-data1 & syn-data2 \\
\hline Spatially Regularized Fused Lasso & 0 & $1 \%$ \\
K-means Clustering & 0 & $10.90 \%$ \\
Modularity detection & 0 & $11.30 \%$ \\
\hline
\end{tabular}

Table 4: Average Error Percentage from Synthetics Data Sets

\begin{tabular}{lll}
\hline & syn-data1 & syn-data2 \\
\hline Spatially Regularized Fused Lasso & $0.10 \%$ & $2.9 \%$ \\
K-means Clustering & $0.11 \%$ & $11.83 \%$ \\
Modularity detection & $0.09 \%$ & $12.47 \%$ \\
\hline
\end{tabular}

mation, it was difficult to deal with noise that was not normally distributed and the outliers can not be eliminated. As a result, a spatially continuous parcellation could not be guaranteed. Nevertheless, the spatially regularized fused lasso model with the spatial regularization term can provide reliable parcellation with spatially continuous and functionally consistent subROIs.

\subsection{Real FMRI Data Set}

\subsubsection{Data Description}

9 healthy subjects were recruited from the Pacific Parkinson's Research Centre (PPRC)/Movement Disorders Clinic at the University of British Columbia (UBC). All experiments were approved by the Ethics Board at UBC, and all subjects provided informed consent prior to experiment participation. During the experiment, all subjects were required to lie on their back in the scanner 


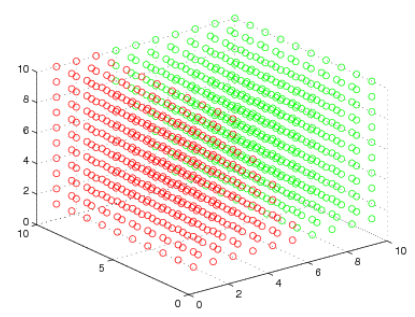

(a)

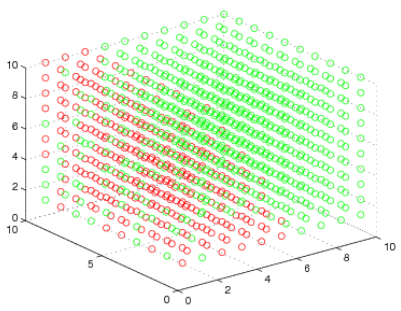

(c)

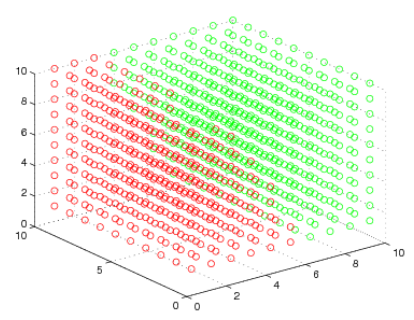

(b)

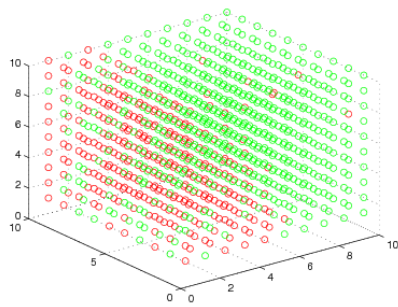

(d)

Figure 4: One example of parcellation results from syn-data2. (a) ground truth. (b) results of the proposed spatially regularized fused lasso algorithm. (c) results of the k-means clustering. (d) results of the modularity detection.

with their eyes closed and keep awake.

MRI data were collected from a Philips Achieva 3.0 T scanner (Philips, Best, Netherlands) equipped with a headcoil. A whole brain three-dimensional T1weighted image consisting of 170 axial slices with high resolution were acquired to facilitate the anatomical localization for each individual. Blood oxygenation level-dependent (BOLD) contrast echo-planar (EPI) T2*-weighted images were taken with the following specifications: repetition time $1985 \mathrm{~ms}$, echo time 37 ms, flip angle $90^{\circ}$, field of view (FOV) $240.00 \mathrm{~mm}$, matrix size $128 \times 128$, and pixel size $1.9 \mathrm{~mm} \times 1.9 \mathrm{~mm}$. The duration of each functional run was $8 \mathrm{~min}$ during which we obtained 240 time points of 36 axial slices with $3 \mathrm{~mm}$ thickness and $1 \mathrm{~mm}$ gap thickness. The FOV was set to include the cerebellum ventrally and the dorsal surface of the brain. 


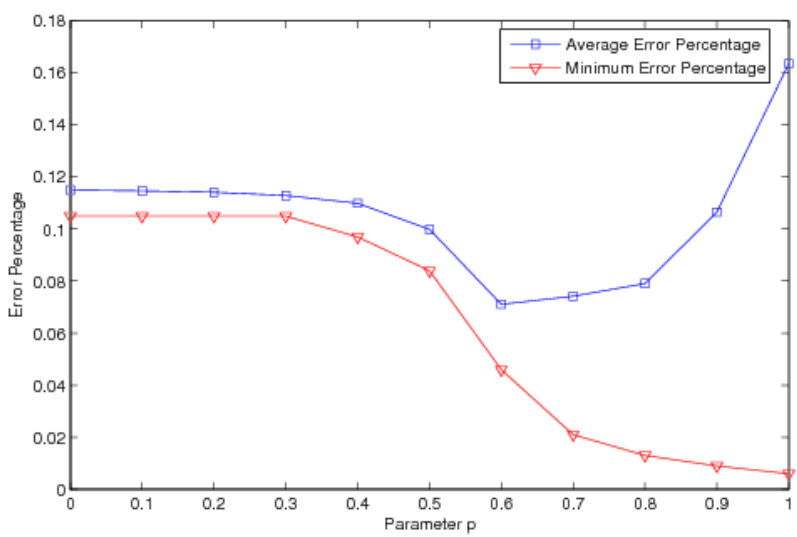

Figure 5: Simulation results from spatially regularized k-means methods with different parameter $p$ chosen. The results are shown from average error rate perspective and also minimum error rate perspective. The upper line (blue) shows the average error rate changing with spatial regularization parameter $p$, while the lower line (red) shows the minimum error rate trending.

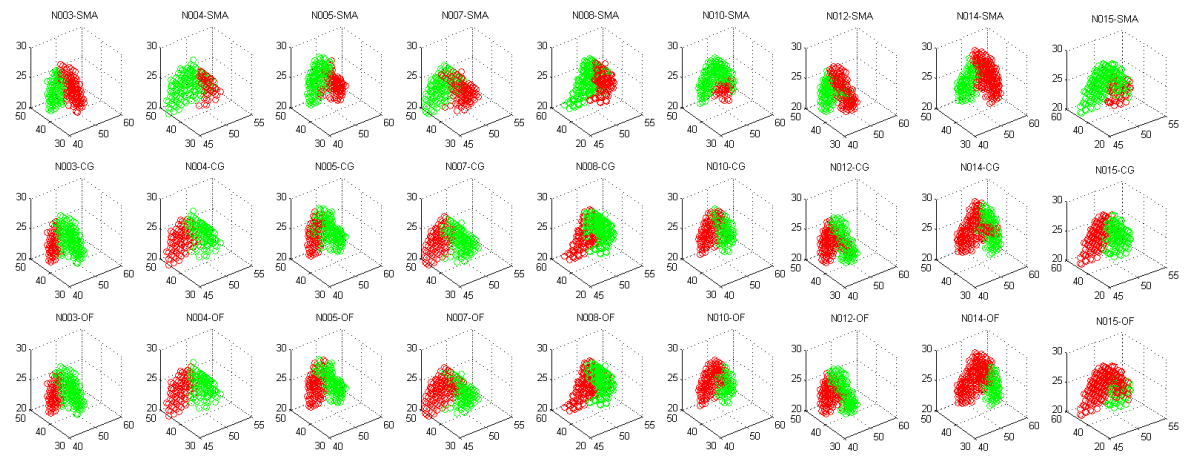

Figure 6: Initial parcellation results for 9 normal subjects according to 3 reference ROIs. Each column shows the results from the same subject according to 3 different reference ROIs, while each row represents results from the same reference ROI across different subjects. In each scatter plot, voxels are shown as small circles with different colors representing groups, and the red color demonstrates significantly stronger correlations. It is worth noting that in our fMRI data, as a result of not using the common spatial template, the spatial coordination of the putamen region changes from subject to subject. 
After acquiring the raw fMRI data, we utilized our own optimized preprocessing pipeline of the fMRI data[29, which includes SPM-based slice timing correction, isotropic reslicing (based on algorithms generated by our team), SPMbased motion correction and FSL-derived registration. 54 regions-of-interest (ROIs) were derived from the open source Freesurfer program (http://freesurfer.net/) for time course extraction. Rather than warping brain images into a common template for connectivity analysis, all data analysis were done on the unwarped images (in the native space) on a subject-by-subject basis.

\subsection{Region of Interest(ROI) Extraction}

We employed the open source Freesurfer program and the available standard atlases for cortical parcellation and subcortical segmentation. Freesurfer uses a high-dimensional registration process by utilizing probabilistic atlases for cortical and subcortical labelling. The labels are then propagated back to the subjects native space by relying on the template label and the subjects transformed voxel value obtained from T1-weighted structural scans. Based on our experience, this ROI-based segmentation method is superior to manually-drawn ROIs and voxel-based method as it minimises registration error particularly in subcortical brain regions such as the putamen.

For highly accurate structural analyses of subcortical structures, we have in the past used Large Deformation Diffeomorphic Metric Mapping (LDDMM) [28]. However, we note that we are using the Freesurfer segmentation of the high-resolution structural scans $(1 \mathrm{x} 1 \mathrm{x} 1 \mathrm{~mm})$ to provide ROI-based binary masks to the relatively low-resolution fMRI scans $(3 \times 3 \times 3 \mathrm{~mm})$. We have found the Freesufer segmentations well-suited for this purpose. The extracted ROIs are visually checked by experienced neurologists if needed.

Table 5 lists all four of the regions-of-interest (ROIs) extracted from both sides of the brain. For simplicity, the right column contains only Freesurfer and Human Motor Area Template (HMAT) labels from the left side of the brain. HMAT labels are in bold. In this study, the orbitofrontal gyrus (OF) is defined by both the medial and lateral orbital frontal cortices, the cingulate gyrus in- 
Table 5: List of ROIs Used in This Paper

\begin{tabular}{|l|l|}
\hline Regions of Interest (ROI) & Freesurfer and HMAT labels \\
\hline Sensorimotor Area (SMA) & ctx-lh-postcentral, L_S1 L_M1 \\
\hline Cingulate gyrus (CG) & $\begin{array}{l}\text { ctx-lh-caudalanteriorcingulate, } \\
\text { ctx-lh-posteriorcingulate }\end{array}$ \\
\hline Orbitofrontal gyrus (OF) & $\begin{array}{l}\text { ctx-lh-medialorbitofrontal, } \\
\text { ctx-lh-lateralorbitofrontal }\end{array}$ \\
\hline Putamen & Left-Putamen \\
\hline
\end{tabular}

clude the anterior and posterior cingulate gyri, and finally the somatosensory area consists of the primary motor cortex as well as the somatosensory cortex.

\subsubsection{Algorithm Application}

In the real fMRI data application, in this pilot study, we only applied our algorithm on the left hemisphere. We choose OF, CG and SMA as reference ROIs as discussed before, to separate the putamen region into two functional striatal subROIs - the DLS and DMS. We assumed the motor control loops of the DLS and DMS were independent. Before performing any analysis, we first removed the global mean artifact in the putamen region and all three reference ROIs through linear regression. Then, in order to exploit the partial correlation between each reference ROI and the putamen region, we also removed artifacts from the SMA in the OF and CG and artifacts from the CG and OF in the SMA using the same method. The DLS is assumed to be functionally connected to the SMA. Therefore, we take SMA as a reference ROI to define the DLS in the putamen region. In order to determine DLS, we calculated the correlation coefficients between each voxel contained in the putamen and mean time course of SMA. After transferring correlation coefficients to Fisher's Z-statistics, a one-sided Welch's t-test is applied on magnitude of z-statistics to test whether one region has significantly stronger (under 5\% significance level) correlation with the SMA and the region with stronger correlation is defined as the DLS. Similarly, to define the DMS in putamen region, OF and CG are 
selected as the reference ROIs according to prior knowledge. The Graph-Cut algorithm is essential in this case to combine two parcellation results based on connections to the CG and OF. Figure 6 shows initial parcellation results from 9 normal subjects respectively with SMA, CG and OF as the reference ROI. Each point represents one voxel in 3-dimensional coordinates in Figure 6. Red and green colors denote two functional subROIs respectively with red representing stronger correlation. Figure 7 demonstrates the final definition of the DLS and DMS from 9 normal subjects with red representing the DMS and green representing the DLS. In Figure 8, we showed our parcellation result of one subject (N003) with registration on the structural image.

\subsubsection{Performance and Group Consistency Evaluation}

Since we have no access to the ground truth of parcellation of real fMRI data, we have to indirectly validate our results anatomically or functionally. Firstly, under the assumption that functional subROIs exist in the putamen region, it is reasonable to assume that the anatomical boundaries across normal subjects are stable. However, since our data do not use a common spatial template for all subjects, spatial coordination of the putamen region changes subtly from subject to subject. As a result, it is not easy to evaluate the consistency of anatomical segmentation. The final parcellation results in Figure 7 show that the DMS region (red) is consistently located on the right side of the putamen region while all DLS points (green) are on the left side. Our results show that clear boundaries are consistently observed in normal subjects, however the specific positions of the boundaries vary from subject to subject probably due to the inter-subject variability. In addition, since our proposed algorithm already incorporates prior knowledge (as shown in Figure 1) into the optimization process, the definition of the DLS and DMS is strongly consistent with prior knowledge. The results demonstrate that we could obtain spatially continuous and functionally consistent subROIs and suggests that the proposed method could provide a promising way for brain parcellation.

The adaptive changes of parameters of the proposed algorithm is shown in 

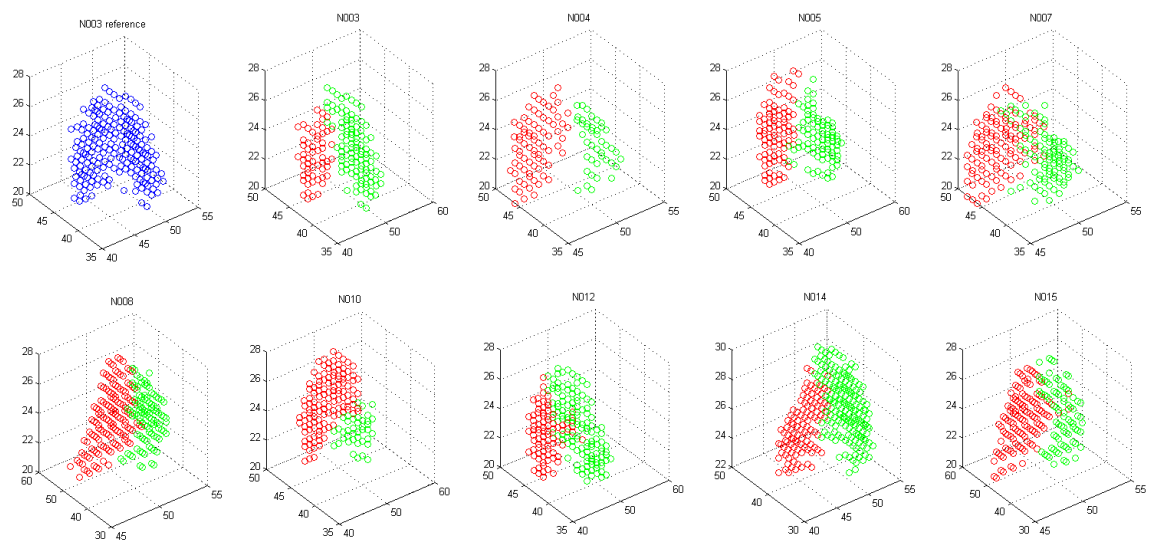

Figure 7: Final parcellation results from 9 normal subjects, where red points represent DMS region defined by combining initial results from CG and OF, green dots represent DLS region defined by initial results from SMA. Since DLS and DMS are defined by different parcellation region, the two groups could be totally spatially separated or overlapped. For reference, the first graph(upper left) shows the full putamen region in subject N003 without any parcellation. Note that although the original accurate anatomical definition of putamen region may change subtly from subject to subject, their geometrical shapes remain almost the same.

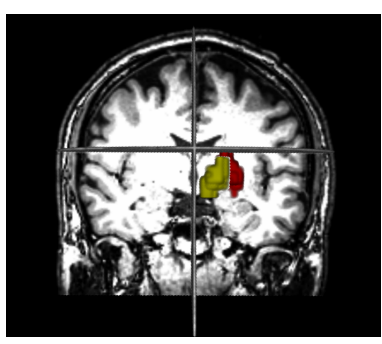

(a) Coronal

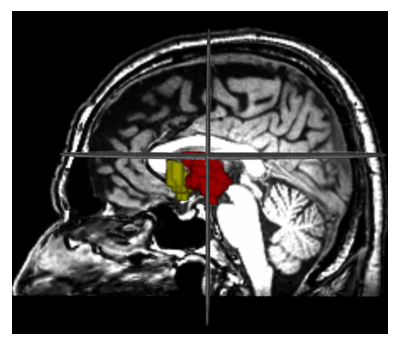

(b) Saggital

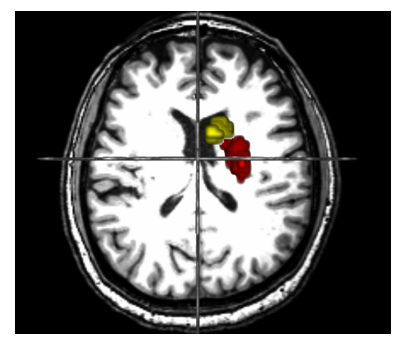

(c) Axial

Figure 8: One example of parcellation results in structural image from N003. Here the red region represents the DLS while the yellow region shows the DMS.

Figure 9 with the red line representing the number of groups, green and blue lines representing tuning parameters. Recall that in our algorithm, if the number of groups could not be reduced, we will increase the penalty parameters. It's worth noting that in the last few iterations, $\gamma$ and $\lambda$ increase dramatically since the algorithm is trying to combine those isolated outliers into the groups which 


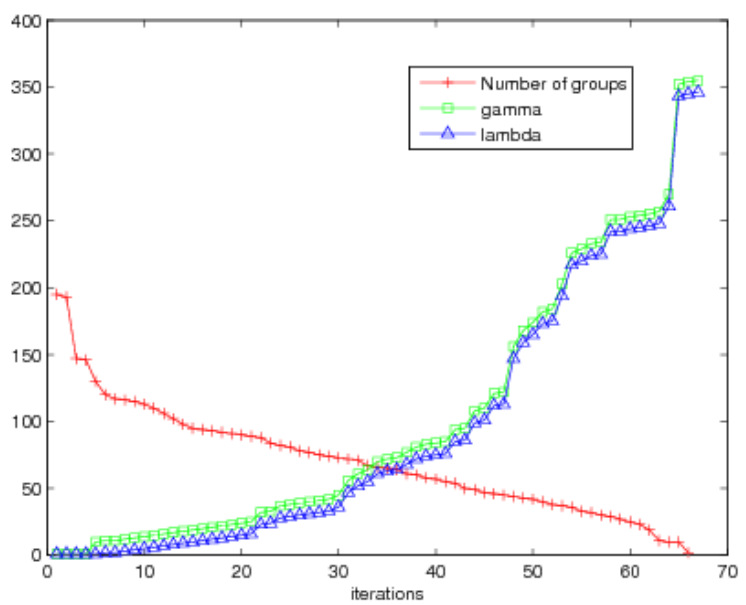

Figure 9: The iteration process in one parcellation (parcellating putamen according to SMA of one normal subject). The red line shows the change of number of groups in each iteration, the green and blue lines represent the change of the spatially regularized lasso parameter $\gamma$ and the normal lasso parameter $\lambda$ respectively.

demands large tuning parameters in the last several iterations,.

\section{Discussion and Conclusion}

In this paper, we presented a novel framework for parcellating one brain ROI into distinct functional subROIs using fMRI based functional connectivity patterns with other reference ROIs. The proposed approach applies the spatially regularized fused lasso model to obtain functional connectivity between voxels in the task ROI with other reference ROIs. With the learned connectivity patterns, we grouped adjacent voxels with similar connectivity weights together. Then, groups containing similar connectivity adjacent voxels will be treated as new voxels and the process repeated until the expected number of subROIs are obtained as described in Table 1. Lastly, the Graph-Cut algorithm is applied to assign voxels by combining the parcellation results from several reference ROIs.

Several parameters are included in this proposed method. First, the parameter $\lambda$ controls the sparsity of elements in the connectivity coefficient vector, $\beta$. 
This parameter can be set to 0 in order to consider only the sparsity difference. Assigning a larger $\lambda$ to the algorithm does mean a higher tolerance of noise, however, it can also increase the possibility of merging all voxels into one group. In our simulation on synthetic data, we set it to 1 while on real dataset, it was set to 10. Second, the parameter $\gamma$ controls the sparsity of difference between successive elements in the connectivity vector $\beta$. It is important to note that $\gamma$ can not be set as 0 , as otherwise, the algorithm cannot converge. If the initial $\gamma$ value is set too high, too many voxels will be merged in the first step, resulting in possibly biased final results. In our experiment, we set $\gamma$ to 10 on synthetic dataset whereas on real fMRI dataset, due to the existence of different spatial noises in different subjects, we adjusted the parameter on a subject by subject basis. An appropriate choice of this parameter is recognized by well-balanced grouping results; there should not be a single group that contains less than 10 voxels or be located in the middle of other groups. The third parameter is the merging precision parameter, $\delta$, which as its name suggests, controls the precision of merging two adjacent voxels with similar connectivity weights. If $\delta$ is set too small, it risks merging of all voxels into only one group whereas a large $\delta$ would make it difficult for the algorithm to converge. In our applications on synthetic and real data, we set the initial value of $\delta$ to $10^{4}$. It is worth noting that even though we have adaptively adjusted all parameters, we still have to choose the initial value carefully. Inappropriate choices of initial parameters may lead to ill-balanced results.

We have tested the proposed algorithm on synthetic datasets and compared the result with two other state-of-the-art methods. It is shown that the proposed algorithm is comparable with these algorithms in general. In particular, the proposed method is better-suited to deal with cases which do not have normally distributed noise or datasets corrupted with outliers. In a real fMRI data, we applied the proposed framework to nine healthy subjects in order to define two functional subROIs of the putamen known as the DLS and DMS. The results are consistent with prior knowledge.

We are motivated to develop this novel method to address the need in neurol- 
ogy and neuroscience research, i.e., to parcellate the putamen into two functional parts (DLS and DMS) based on its connectivity with three other reference ROIs. The proposed algorithm is applicable to a set of neurological problems where there exist one task ROI to be parcellated into several functional subROIs and a few reference ROIs that have different connectivity patterns with these functional subROIs. The proposed algorithm could be applied to both task-related data sets as well as resting fMRI data sets. Furthermore, with suitable parameter adjustments, we do not require the sophisticated signal denoising procedure. However, it is worth mentioning that, although our algorithm can better deal with outliers compared with other two methods, outlier detection and removing is still essential for many connectivity analysis problems.

Since the proposed method is based on functional connectivity, people may argue that the results for subsequent connectivity analysis may be biased. We could reduce such risks of bias by separating time signals into two parts (e.g., the odd-indexed points as one part and the even-indexed points as the other part), and use one part to obtain consistent parcellation results and then use the other part of data for further connectivity analysis. We could also benefit from this two-part idea for the validation of the parcellation results: We can parcellate the functional ROI with one part of the fMRI time-series signals and validate the functional parcellations with the other part of the fMRI signals. Consistency measures such as the discrete entropy can be employed to evaluate the consistency of the parcellation results from using different parts of the fMRI signals.

The proposed method can be applied to study geriatric and neuro-diseased populations. The extracted functional subROIs themselves are of great interest to study the influence of aging and neurodegenerative diseases. In future study, we plan to use the proposed method to investigate the changes of DLS subROIs in subjects with Parkinson's disease, where it may serve as a potential biomarker of Parkinson's disease. 


\section{Acknowledgement}

This work was supported by Natural Sciences and Engineering Research Council of Canada (NSERC) and Canadian Institutes of Health Research (CIHR) and National Natural Science Foundation of China (Grant No. 81571760 and $61501164)$.

\section{References}

[1] M. J. McKeown, S. Makeig, G. G. Brown, T.-P. Jung, S. S. Kindermann, A. J. Bell, T. J. Sejnowski, Analysis of fmri data by blind separation into independent spatial components, Tech. rep., DTIC Document (1997).

[2] K. J. Friston, L. Harrison, W. Penny, Dynamic causal modelling, Neuroimage 19 (4) (2003) 1273-1302.

[3] A. Mclntosh, F. Gonzalez-Lima, Structural equation modeling and its application to network analysis in functional brain imaging, Human Brain Mapping 2 (1-2) (1994) 2-22.

[4] M. P. Van Den Heuvel, H. E. Hulshoff Pol, Exploring the brain network: a review on resting-state fmri functional connectivity, European Neuropsychopharmacology 20 (8) (2010) 519-534.

[5] A. Liu, X. Chen, M. J. McKeown, Z. J. Wang, A sticky weighted regression model for time-varying resting state brain connectivity estimation, IEEE Transactions on Biomedical Engineering (2014) (in press).

[6] A. Liu, X. Chen, Z. J. Wang, Q. Xu, S. Appel-Cresswell, M. J. McKeown, A genetically-informed, group fmri connectivity modeling approach: application to schizophrenia, IEEE TRANSACTIONS ON BIOMEDICAL ENGINEERING 61 (3) (2013) 946 - 956.

[7] A. Liu, J. Li, Z. J. Wang, M. J. McKeown, A computationally efficient, exploratory approach to brain connectivity incorporating false discovery 
rate control, a priori knowledge, and group inference, Computational and mathematical methods in medicine 2012.

[8] A. L. Cohen, D. A. Fair, N. U. Dosenbach, F. M. Miezin, D. Dierker, D. C. Van Essen, B. L. Schlaggar, S. E. Petersen, Defining functional areas in individual human brains using resting functional connectivity mri, Neuroimage 41 (1) (2008) 45-57.

[9] J. Rademacher, V. Caviness, H. Steinmetz, A. Galaburda, Topographical variation of the human primary cortices: implications for neuroimaging, brain mapping, and neurobiology, Cerebral Cortex 3 (4) (1993) 313-329.

[10] M. Beckmann, H. Johansen-Berg, M. F. Rushworth, Connectivity-based parcellation of human cingulate cortex and its relation to functional specialization, The Journal of neuroscience 29 (4) (2009) 1175-1190.

[11] R. Baumgartner, G. Scarth, C. Teichtmeister, R. Somorjai, E. Moser, Fuzzy clustering of gradient-echo functional mri in the human visual cortex. part i: Reproducibility, Journal of Magnetic Resonance Imaging 7 (6) (1997) 1094-1101.

[12] T. Kahnt, L. J. Chang, S. Q. Park, J. Heinzle, J.-D. Haynes, Connectivitybased parcellation of the human orbitofrontal cortex, The Journal of Neuroscience 32 (18) (2012) 6240-6250.

[13] L. Wang, Q. Liu, H. Li, D. Hu, Functional connectivity-based parcellation of human medial frontal cortex via maximum margin clustering, in: Intelligent Science and Intelligent Data Engineering, Springer, 2013, pp. 306-312.

[14] X. Shen, X. Papademetris, R. T. Constable, Graph-theory based parcellation of functional subunits in the brain from resting-state fmri data, Neuroimage 50 (3) (2010) 1027-1035.

[15] K. A. Barnes, A. L. Cohen, J. D. Power, S. M. Nelson, Y. B. Dosenbach, F. M. Miezin, S. E. Petersen, B. L. Schlaggar, Identifying basal ganglia di- 
visions in individuals using resting-state functional connectivity mri, Frontiers in systems neuroscience 4(2010).

[16] R. Tibshirani, M. Saunders, S. Rosset, J. Zhu, K. Knight, Sparsity and smoothness via the fused lasso, Journal of the Royal Statistical Society: Series B (Statistical Methodology) 67 (1) (2005) 91-108.

[17] P. Griffiths, R. Perry, A. Crossman, A detailed anatomical analysis of neurotransmitter receptors in the putamen and caudate in parkinson's disease and alzheimer's disease, Neuroscience letters 169 (1) (1994) 68-72.

[18] A. J. Gruber, R. J. McDonald, Context, emotion, and the strategic pursuit of goals: interactions among multiple brain systems controlling motivated behavior, Frontiers in behavioral neuroscience 6 (2012).

[19] P. Redgrave, M. Rodriguez, Y. Smith, M. C. Rodriguez-Oroz, S. Lehericy, H. Bergman, Y. Agid, M. R. DeLong, J. A. Obeso, Goal-directed and habitual control in the basal ganglia: implications for parkinson's disease, Nature Reviews Neuroscience 11 (11) (2010) 760-772.

[20] R. Tibshirani, Regression shrinkage and selection via the lasso, Journal of the Royal Statistical Society. Series B (Methodological) (1996) 267-288.

[21] G. Tononi, A. R. McIntosh, D. P. Russell, G. M. Edelman, Functional clustering: identifying strongly interactive brain regions in neuroimaging data, Neuroimage 7 (2) (1998) 133-149.

[22] S. Kim, K.-A. Sohn, E. P. Xing, A multivariate regression approach to association analysis of a quantitative trait network, Bioinformatics 25 (12) (2009) i204-i212.

[23] X. Chen, Q. Lin, S. Kim, J. G. Carbonell, E. P. Xing, et al., Smoothing proximal gradient method for general structured sparse regression, The Annals of Applied Statistics 6 (2) (2012) 719-752. 
[24] C. H. Papadimitriou, K. Steiglitz, Combinatorial optimization: algorithms and complexity, Courier Dover Publications, 1998.

[25] Y. Boykov, O. Veksler, R. Zabih, Fast approximate energy minimization via graph cuts, Pattern Analysis and Machine Intelligence, IEEE Transactions on 23 (11) (2001) 1222-1239.

[26] Y. Boykov, V. Kolmogorov, An experimental comparison of min-cut/maxflow algorithms for energy minimization in vision, Pattern Analysis and Machine Intelligence, IEEE Transactions on 26 (9) (2004) 1124-1137.

[27] V. Kolmogorov, R. Zabin, What energy functions can be minimized via graph cuts?, Pattern Analysis and Machine Intelligence, IEEE Transactions on 26 (2) (2004) 147-159.

[28] F. Deleus, M. M. Van Hulle, A connectivity-based method for defining regions-of-interest in fmri data, Image Processing, IEEE Transactions on 18 (8) (2009) 1760-1771.

[29] Chen, Jingyun, et al. "Freesurfer-initialized large deformation diffeomorphic metric mapping with application to Parkinson's disease." SPIE Medical Imaging. International Society for Optics and Photonics, 2009. 